\title{
Quantifying three-dimensional optic axis using polarization-sensitive optical coherence tomography
}

Chao J. Liu

Adam J. Black

Hui Wang

Taner Akkin 


\section{Quantifying three- dimensional optic axis using polarization- sensitive optical coherence tomography}

\author{
Chao J. Liu, Adam J. Black, Hui Wang, and \\ Taner Akkin* \\ University of Minnesota, Department of Biomedical Engineering, \\ 312 Church Street SE, Minneapolis, Minnesota 55455, United States
}

\begin{abstract}
The optic axis of birefringent samples indicates the direction of optical anisotropy, which should be described in three-dimensional (3-D) space. We present a method to quantify the complete 3-D optic axis orientation calculated from in-plane optic axis measurements from a polarization-sensitive optical coherence tomography system. The in-plane axis orientations with different illumination angles allow the calculation of the necessary polar angle. The method then provides the information to produce the actual birefringence. The method and results from a biological sample are presented. ๑ 2016 Society of PhotoOptical Instrumentation Engineers (SPIE) [DOI: 10.1117/1.JBO.21.7.070501]
\end{abstract}

Keywords: optical coherence tomography; polarization; axis measurement.

Paper 160143LRR received Mar. 9, 2016; accepted for publication Jun. 16, 2016; published online Jul. 7, 2016.

Optical coherence tomography (OCT) produces depth-resolved images of tissue microstructure. ${ }^{1}$ In addition to the conventional reflectivity contrast, polarization-sensitive OCT (PS-OCT) provides retardance and optic axis orientation contrasts that originate from tissue birefringence. ${ }^{2}$ Birefringence, the primary source of the polarization contrasts, is an optical measure of structural anisotropy. It is defined as the difference between the refractive indices for light polarized parallel and perpendicular to the optic axis. The polarization state of light traveling in birefringent tissue changes due to a delay between the orthogonal polarization components. The delay, known as retardance, is equivalent to the birefringence multiplied by the distance the light travels, and it can be represented by phase as one wavelength corresponds to $360 \mathrm{deg}$. It is important to realize that polarization-based optical systems relying on one illumination angle measure the "apparent birefringence" light encounters rather than the "true birefringence." orientation is defined in a plane $(x y)$ orthogonal to the direction of light propagation $(z)$ and can only yield a two-dimensional (2-D) projection of the axis of anisotropy.
Both azimuthal and polar angles are required to describe tissue axis orientation in three dimension. Polarimetric studies have been presented for describing the three-dimensional (3-D) optic axis orientation and intrinsic retardance., ${ }^{4,5}$ Computational methods based on OCT have been reported to extract 3-D orientation. ${ }^{6,7}$ Using retardance measurements (band spacing), a variant-incident angle PS-OCT has been reported to determine the polar angle of the optic axis of a uniaxial birefringent sample (tendon) as well as the true birefringence. ${ }^{8}$ This method assumes or measures the ordinary refractive index and calculates the extraordinary refractive index and the polar angle using an equation derived from the definition of apparent birefringence. While the band spacing of retardance is accessible for highly birefringent tissue, a complete band may not be visible for weakly birefringent tissue for which the slope of retardance with respect to depth can be estimated. ${ }^{9}$

In this letter, we propose a method to quantify the 3-D axis orientation of anisotropic tissues from the in-plane (2-D) optic axis orientations measured with different incident angles. While the axis orientation in a single plane can provide the azimuth of anisotropy, the polar angle can be calculated by using the retrieval method presented here. This method requires the axis orientation information in an additional plane by varying the incident angle. We use a custom polarization-maintaining fiber (PMF) based PS-OCT system and introduced calibration paths to dynamically remove the arbitrary offset of the in-plane axis orientation measurements of such systems. Without the calibration, the axis measurement is a relative measure in an unknown coordinate system that is potentially time varying. The polar angle retrieval method is tested by using a rat muscle sample orientated at different azimuthal and polar angles. After finding the polar angle, the actual birefringence of the sample is quantified.

Single mode non-PMFs ${ }^{10}$ and $\mathrm{PMF}^{11}$ have been used to realize PS-OCT. On one hand, polarization state in non-PMF changes due to a random birefringence; on the other hand, PMF preserves the linear states propagating in its orthogonal polarization channels. PMF-based PS-OCT has several advantages, but optical path lengths of PMF channels can slightly vary with environmental factors (fiber movement and temperature change) inducing an arbitrary offset for the optic axis orientation. The offset can be measured and removed by imaging a retarder with known axis placed next to the sample, ${ }^{12}$ which may be hard to implement under a high-NA lens due to a short-working distance. Instead, we used calibration paths in this study. Calibration has also been reported for non-PMF-based PS-OCT to determine absolute fast axis orientation. ${ }^{13}$

Figure 1 shows the optical system. Our earlier publication reports the main system (sensitivity: $98.6 \mathrm{~dB}$; integration time: $50 \mu \mathrm{s}) .{ }^{14}$ Here, calibration units are added to the reference and sample arms to compensate for the arbitrary offset in the optic axis orientation measurement, and the sample was mounted on a rotational stage to vary the incident angle. Briefly, light $(\lambda=840 \mathrm{~nm}, \Delta \lambda=50 \mathrm{~nm})$ is polarized and coupled into a PMF channel. A PMF-based coupler splits the light into the reference and sample arms. In the reference arm, a quarter-wave plate (QWP) is orientated at 22.5 deg to return equal light levels to both PMF channels. The QWP in the sample arm is oriented at $45 \mathrm{deg}$ to the incoming linear state, so that circularly polarized light is launched onto the sample. Light returning from the 


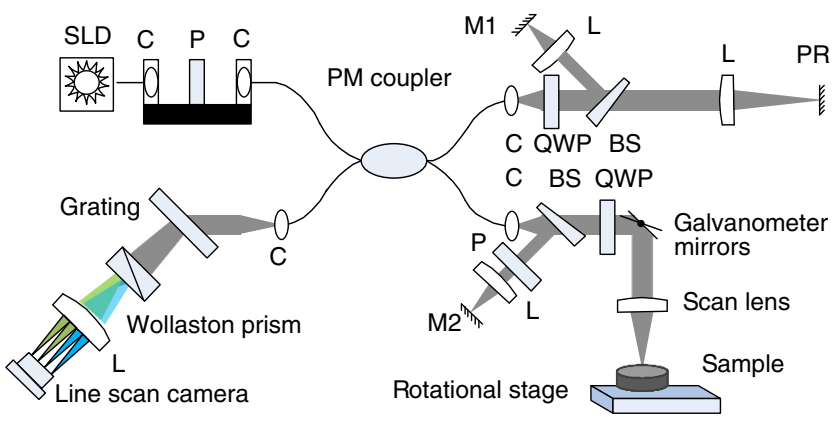

Fig. 1 Optical system. SLD, superluminescent diode; P, polarizer; C, collimator; L, lens; PR, partial reflector (glass wedge); M1, M2: mirror; QWP, quarter wave plate; BS, beam sampler. Coupler is with PMF.

sample and the reference reflector (PR in Fig. 1) interferes in the PMF coupler and forms the basis of PS-OCT imaging. A custom spectrometer simultaneously records interference patterns on two spectra corresponding to the PMF channels. The spectrometer is based on a single CMOS line scan camera, on which two spectra are focused side-by-side.

The two spectra are extracted and interpolated to represent the interference-related spectral oscillations that are linear in $k$ (wave number) space. An inverse Fourier transform is applied to each spectrum to obtain the complex depth profiles (A-line) that are in the form of $A_{1,2}(z) \exp \left\{i \phi_{1,2}(z)\right\}$, where $A$ and $\phi$ denote the amplitude and phase as a function of depth $z$ and the subscripts represent the polarization channels. The reflectivity $R(z)$, phase retardance $\delta(z)$, and relative axis orientation $\theta^{\prime}(z)$ are derived from the magnitudes and phases of the complex depth profiles ${ }^{15}$ as $R(z) \propto A_{1}(z)^{2}+A_{2}(z)^{2}, \delta(z)=$ $\tan ^{-1}\left[A_{1}(z) / A_{2}(z)\right], \quad$ and $\quad \theta^{\prime}(z)=\left\{\phi_{1}(z)-\phi_{2}(z)\right\} / 2+\phi_{o}$. Note that $\phi_{o}$ is the aforementioned arbitrary offset, which is removed from the measurement described as follows.

The reference arm after the QWP consists of a beam sampler that diverts $\sim 1 \%$ of the reference light to the calibration unit that includes a lens and a mirror (M1). The sample arm prior to the QWP also consists of a similar beam sampler for providing light to the second calibration unit, which is formed by a $45 \mathrm{deg}$ polarizer, a lens, and a mirror (M2). The polarization-dependent loss is minimized by aligning the beam samplers at nearly normal incidence. ${ }^{16}$ Each calibration unit couples equal power back to the PMF channels. Light from these calibration units interfere with each other in the coupler to generate two coherence functions, one on the main channel and the other on the cross channel, resulting in a surface in the depth profile. The depth location of this surface is adjusted by the mirror (M1, M2) locations, and it is typically located below the imaging area used for the sample. The phase information from the surface dynamically provides the arbitrary offset $\phi_{o}$, which is then subtracted from the sample's relative axis orientation data. Thus, the absolute axis orientation is obtained by $\theta(z)=\theta^{\prime}(z)-\phi_{o}$.

As the offset can be time dependent, we measured optic axis orientation of a known retarder at different time points. First, the retarder was orientated at $20 \mathrm{deg}$. Figure 2(a) shows the relative axis orientation measurement (rectangles) fluctuating between $\sim 40 \mathrm{deg}$ and $\sim 180 \mathrm{deg}$ within a total of $\sim 5 \mathrm{~h}$ measurement time. The offset measurement (circles) from the calibration surface varied with the same trend; therefore, its removal from the relative axis orientation measurement successfully revealed the absolute axis orientation (triangles). The mean and standard deviation values of the orientation measurement are $19.4 \mathrm{deg}$

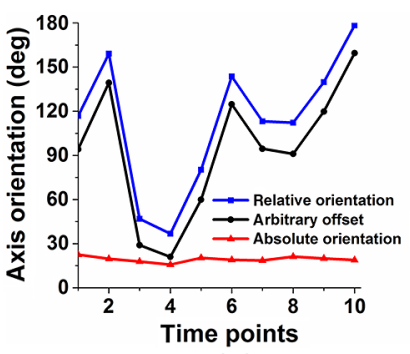

(a)

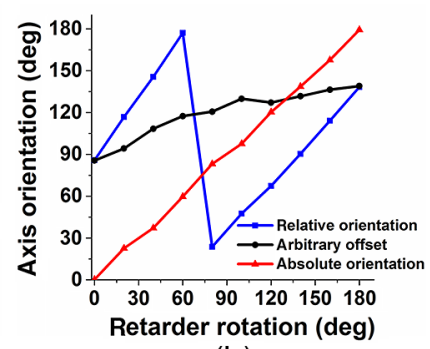

(b)
Fig. 2 (a) Measurement and removal of arbitrary offset for absolute axis orientation, measurement time: $\sim 5 \mathrm{~h}$. (b) Axis orientation of a known retarder with 20 -deg rotational steps from 0 deg to 180 deg.

and $1.9 \mathrm{deg}$, respectively. Then, the known retarder was rotated from 0 deg to $180 \mathrm{deg}$ in $20 \mathrm{deg}$ increments. Figure 2(b) shows the measured relative axis orientations (rectangles), the offset values (circles), and the corresponding absolute axis orientations (triangles). The absolute axis orientation values increase from $0.2 \mathrm{deg}$ to $179.3 \mathrm{deg}$ with a slope of $\sim 0.99$ (linear fitting), as expected.

In a spherical coordinate system, the polar angle $\Psi$ and the azimuthal angle $\Phi$ describe the axis orientation of a birefringent fiber tract in 3-D. Considering a normal incidence of light along the $z$-axis, the azimuthal angle can be determined from the first axis orientation measurement $\theta_{1}$ thus, $\Phi=\theta_{1}$. The polar angle, on the other hand, requires the axis measurement in another plane selected by varying the incident angle or by rotating the sample with respect to the light. Rotating the sample by an angle $\Omega_{x}$ about the $x$-axis, we measure a second optic axis orientation $\theta_{2}$, which is a projection of the 3-D axis to the plane $\left(x y^{\prime}\right)$. Note that the selected planes are always orthogonal to the beam direction. By using the definitions of the Cartesian and spherical coordinates, a rotation matrix and trigonometric identities in a right-handed coordinate system, the polar angle $\Psi$ can be calculated by the following equation:

$\Psi=\arctan \left(\frac{\sin \Omega_{x}}{\sin \theta_{1} \cos \Omega_{x}-\cos \theta_{1} \tan \theta_{2}}\right)$.

Following the right-hand rule, positive $\Omega_{x}$ means counter-clockwise rotation. Note that the inclination angle is simply calculated by $\alpha=90 \mathrm{deg}-\Psi$.

The polar angle $(\Psi)$ calculation from Eq. (1) is simulated by setting the first in-plane axis orientation $\left(\theta_{1}=0 \mathrm{deg}, 30 \mathrm{deg}\right.$, $60 \mathrm{deg}, 89 \mathrm{deg})$ and the rotational angle $\left(\Omega_{x}=30 \mathrm{deg}\right)$. Figure 3 shows $\Psi$ as a function of the second axis measurement $\left(\theta_{2}\right)$. While the equation works well for $\theta_{1}=0 \mathrm{deg}, 30 \mathrm{deg}$, and $60 \mathrm{deg}$ (smoother curves), the sharp rise for $\theta_{1}=89 \mathrm{deg}$ is concerning as the noise on $\theta$ measurements would result in unreliable $\Psi$ values. Therefore, Eq. (1) is not sufficient where the fiber tracts run on the $y z$-plane, because the rotation $\Omega_{x}$ should not alter the measured in-plane orientations $\left(\theta_{1} \cong \theta_{2} \cong 90 \mathrm{deg}\right)$.

To test the polar angle retrieval method, we used a uniaxial segment of a rat biceps femoris muscle. The alignment of the muscle fibers appeared to be along one direction under a dissection microscope. A polar angle of $-60 \mathrm{deg}$ was created by placing the muscle sample on a right-triangular prism. The prism was mounted on a rotational stage to vary the angle of incidence. To define the 0 deg axis orientation, the tissue long axis should be aligned with or projected onto the $x$-axis. We measured the axis orientations (in $x y$ - and $x y^{\prime}$-planes) with two different 


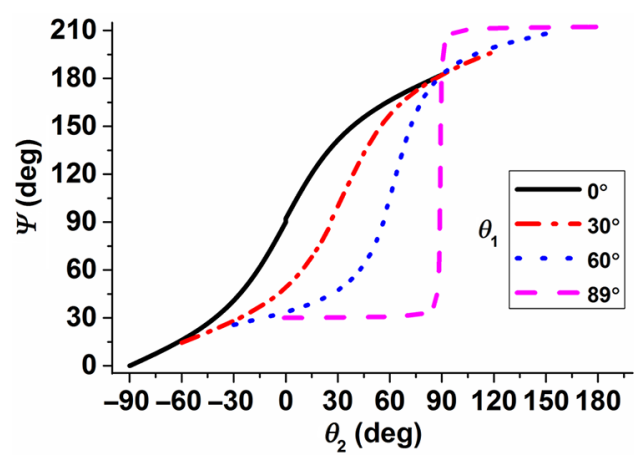

Fig. 3 Polar angle $\Psi$ versus axis orientation in the second plane $\theta_{2}$. $\Omega_{X}$ is set to $30 \mathrm{deg}$. The curves are produced from Eq. (1) by setting the azimuth to $\theta_{1}=0 \mathrm{deg}, 30 \mathrm{deg}, 60 \mathrm{deg}$, and $89 \mathrm{deg}$.

incident angles to test the method for four different azimuths set to $0 \mathrm{deg}, 30 \mathrm{deg}, 60 \mathrm{deg}$, and $90 \mathrm{deg}$. For each measurement, a cross section from a small region $(\sim 0.5 \mathrm{~mm})$ was scanned 20 times and the axis orientation was extracted by taking the peak value of the histogram (bin width: $3 \mathrm{deg}$ ). The mean values of the $\theta$ measurements were used in the calculation of the polar angle. The mean and standard deviations of the $\theta$ measurements are listed in Table 1. For the azimuth set to $0 \mathrm{deg}, 30 \mathrm{deg}$, and $60 \mathrm{deg}$, the calculated polar angles are within a few degrees to each other and close to the set value of $-60 \mathrm{deg}$. The errors may arise from imperfect sample positioning, exclusion of water submersion to minimize the effect of refraction, local axis variation in the tissue sample, and noise in the measurements. The polar angle calculation for $90 \mathrm{deg}$ azimuth is marked as an unreliable case. The reason is that, when $\theta_{1}$ and $\theta_{2}$ measurements both approach $90 \mathrm{deg}$, small variations of $\theta_{1}$ and $\theta_{2}$ measurements cause significant change in $\Psi$ as shown by the sharp rise in Fig. 3.

Table 2 shows the results from the same muscle sample with various rotation angles, $-30 \mathrm{deg} \leq \Omega_{x} \leq 30 \mathrm{deg}$. Azimuth was fixed and measured as $\theta_{1}=-3.3 \mathrm{deg} \pm 1.9 \mathrm{deg}$ at $\Omega_{x}=0 \mathrm{deg}$. After measuring $\theta_{2}$ values for each $\Omega_{x}$, Eq. (1) was used to retrieve the polar angle. Negative (clockwise) and positive (counterclockwise) rotation angles both yielded polar angle values close to the set value $(-60 \mathrm{deg})$.

To test the method for a different polar angle, the sample was mounted on a different platform making $\Psi=-45 \mathrm{deg}$. When $\Omega_{x}=0 \mathrm{deg}, \theta_{1}$ was measured to be $-0.1 \mathrm{deg} \pm 2.2 \mathrm{deg} . \theta_{2}$ values were measured at different rotation angles, $-30 \mathrm{deg} \leq \Omega$ $x \leq 30 \mathrm{deg}$. Then, the corresponding polar angles were calculated. Table 3 demonstrates the results, which are close to the expected value.

Table 1 Polar angle (set to $-60 \mathrm{deg}$ ) of a muscle sample is quantified with $\Omega_{x}=30 \mathrm{deg}$ and different azimuths.

\begin{tabular}{lccc} 
Azimuth (deg) & $\theta_{1}$ (deg) & $\theta_{2}$ (deg) & $\Psi$ [from Eq. (1)] (deg) \\
\hline 0 & $-0.25 \pm 1.3$ & $12.2 \pm 2.2$ & -66.3 \\
30 & $27.8 \pm 1.5$ & $35.5 \pm 1.8$ & -65.7 \\
60 & $53.5 \pm 1.4$ & $56.5 \pm 1.7$ & -68 \\
90 & $86.4 \pm 3.3$ & $87.2 \pm 2.9$ & $-51.6^{\mathrm{a}}$ \\
\hline
\end{tabular}

aUnreliable case.
Table 2 Polar angle (set to $-60 \mathrm{deg}$ ) of a muscle sample is quantified with various rotation angles $\theta_{1}=-3.3 \mathrm{deg} \pm 1.9 \mathrm{deg}$.

\begin{tabular}{lcc}
$\Omega_{X}(\mathrm{deg})$ & $\theta_{2}(\mathrm{deg})$ & $\Psi$ [from Eq. (1)] (deg) \\
\hline-30 & $-21.6 \pm 2$ & -55.4 \\
-20 & $-13.5 \pm 2.5$ & -61.5 \\
-10 & $-7.5 \pm 2.1$ & -66.7 \\
10 & $1.7 \pm 3.5$ & -63.6 \\
20 & $9.5 \pm 3$ & -57.2 \\
30 & $10.4 \pm 3.3$ & -65.1 \\
\hline
\end{tabular}

True birefringence $(\Delta n)$ can be defined as the refractive index difference between the fast and slow fiber axes that occurs at $\alpha=0 \mathrm{deg}$ (equivalently, $\Psi=90 \mathrm{deg}$ ). However, the retardance measurement with an existing inclination is related to apparent birefringence $\left(\Delta n^{\prime}\right)$, which is correlated to true birefringence and inclination as $\Delta n^{\prime}=\Delta n \cos ^{2} \alpha{ }^{3}$ Therefore, the measurements of $\Delta n^{\prime}$ (derivative of retardance) and $\alpha$ (or $\Psi$ ) yield $\Delta n$. The $\Psi=-60 \mathrm{deg}$ dataset resulted in $\Delta n^{\prime}=5.31 \times$ $10^{-4}$ and the calculated polar angle of $-66.3 \mathrm{deg}$ (the first row of Table 1). Correspondingly, the true birefringence is calculated to be $6.33 \times 10^{-4}$, which is close to the birefringence value of $6.74 \times 10^{-4}$ measured at a 0 deg inclination angle.

As mentioned, when $\theta_{1} \cong 90 \mathrm{deg}$, the polar and inclination angles cannot be calculated accurately from $\theta_{1}$ and $\theta_{2}$ measurements due to noise. In this case, the use of Eq. (1) would be problematic, because small variations in $\theta$ measurements would significantly alter the $\Psi$ value (see the sharp rise covering the entire span of $\Psi$ values in Fig. 3). To solve the problem, the sample should be rotated about the $y$-axis $\left(\Omega_{y}\right)$ for the third axis measurement $\left(\theta_{3}\right)$, which is then used with $\theta_{1}$ in an equation

$\Psi=\operatorname{arccot}\left(\frac{\sin \theta_{1}}{\sin \Omega_{y} \tan \theta_{3}}-\frac{\cos \theta_{1}}{\tan \Omega_{y}}\right)$.

To test the polar angle calculation by Eq. (2), $\Psi$ was set to $60 \mathrm{deg}$. The axis orientations in $x y$-plane $\left(\theta_{1}\right)$ and $x^{\prime} y$-plane $\left(\theta_{3}\right.$ with $\left.\Omega_{y}=30 \mathrm{deg}\right)$ were measured for four different azimuths set to $0 \mathrm{deg}, 30 \mathrm{deg}, 60 \mathrm{deg}$, and $90 \mathrm{deg}$. Table 4 shows the results. The unreliable case with Eq. (2) occurs at

Table 3 Polar angle (set to $-45 \mathrm{deg}$ ) of a muscle sample is quantified with various rotation angles $\theta_{1}=-0.1 \mathrm{deg} \pm 2.2 \mathrm{deg}$.

\begin{tabular}{lcc}
$\Omega_{X}(\mathrm{deg})$ & $\theta_{2}(\mathrm{deg})$ & $\Psi$ [from Eq. (1)] (deg) \\
\hline-30 & $-23.5 \pm 2.8$ & -48.8 \\
-20 & $-16.3 \pm 2.2$ & -49.3 \\
-10 & $-8.5 \pm 2.5$ & -48.9 \\
10 & $11 \pm 1.7$ & -42 \\
20 & $19.7 \pm 1.9$ & -43.8 \\
30 & $27.7 \pm 1.8$ & -43.7 \\
\hline
\end{tabular}


Table 4 Polar angle (set to $60 \mathrm{deg}$ ) of a muscle sample is quantified with $\Omega_{y}=30 \mathrm{deg}$ and different azimuths.

\begin{tabular}{lccc} 
Azimuth (deg) & $\theta_{1}(\mathrm{deg})$ & $\theta_{3}(\mathrm{deg})$ & $\Psi$ [from Eq. (2)] (deg) \\
\hline 0 & $1.9 \pm 1.5$ & $3.4 \pm 1.5$ & $-57.8^{\mathrm{a}}$ \\
30 & $30.2 \pm 1.5$ & $24.5 \pm 1.5$ & 54.6 \\
60 & $59.5 \pm 1.8$ & $50.9 \pm 3.1$ & 62.6 \\
90 & $91.9 \pm 1.2$ & $71.2 \pm 3.2$ & 53.6 \\
\hline
\end{tabular}

aUnreliable case.

0 deg azimuth, as expected. The $\Psi$ values with other azimuths are comparable to the set value.

If the fibers are not inclined primarily toward the $z$-axis, the azimuthal angle obtained in the $x y$-plane with the first measurement $\left(\theta_{1}\right)$ can be utilized to determine which equation to use to calculate the polar angle accurately. For $\left|\theta_{1}\right| \leq 45 \mathrm{deg}$, Eq. (1) (with $\Omega_{x}$ and $\theta_{2}$ ) should be chosen to benefit from smoother curves in Fig. 3 (less error due to noise on $\theta$ measurements). Otherwise, Eq. (2) (with $\Omega_{y}$ and $\theta_{3}$ ) should be used because $\Psi$ versus $\theta_{3}$ curves (not shown) would be smoother than $\Psi$ versus $\theta_{2}$ curves.

High inclination angles reduce the apparent birefringence and make the axis orientation measurement with $\theta_{1}$ unreliable. In fact, it is possible that $\theta_{2}$ and $\theta_{3}$ measurements can be more reliable than $\theta_{1}$. To compare the reliability of $\theta$ measurements, the retardance information could be used, as higher retardance is associated with smaller inclination of the axis with respect to the selected plane. Therefore, $\theta_{2}$ and $\theta_{3}$ measurements can be used together for calculating $\Psi$ and representing the axis in 3-D. Although the method can quantify the 3-D axis of uniaxial structures, presence of several axes in a small region will complicate the analysis that requires further development.

When the tissue surface is on the $x y$-plane, the first measurement described here will be taken with normal incidence, which is the typical case for OCT imaging. However, using fixed illumination (along $z$-axis) and rotating the sample can induce refraction at the air-tissue interface that deviates the beam from the incident angle. Submerging the sample into water could reduce the effect of refraction on the polar angle quantification. If the sample surface is fixed and the beam is angled for the measurements, the effect of refraction should be considered as well.

In conclusion, we demonstrated a method to track the axis orientation of birefringent tissue in 3-D space using PS-OCT. Absolute in-plane axis orientations were quantified by dynamically removing an arbitrary offset. The axis measurements in different planes are used to calculate the polar angle completing the orientation information in 3-D. With this information, true birefringence can be quantified. The study has potential to facilitate quantitative imaging and mapping for the structural connections in anisotropic tissues. This includes, e.g., a comprehensive 3-D reconstruction of the brain and cerebellum that supports quantitative analysis on white matter content and circuitry.

\section{Acknowledgments}

The work was supported by research grants from the US National Science Foundation (NSF, CBET-1510674) and the Bob Allison Ataxia Research Center (BAARC) at the University of Minnesota.

\section{References}

1. D. Huang et al., "Optical coherence tomography," Science $\mathbf{2 5 4}$, 1178-1181 (1991).

2. J. F. de Boer et al., "Two-dimensional birefringence imaging in biological tissue by polarization-sensitive optical coherence tomography," Opt. Lett. 22(12), 934-936 (1997).

3. L. Larsen et al., "Polarized light imaging of white matter architecture," Microsc. Res. Tech. 70(10), 851-863 (2007).

4. N. Ortega-Quijano, F. Fanjul-Vélez, and J. L. Arce-Diego, "Polarimetric study of birefringent turbid media with three-dimensional optic axis orientation," Biomed. Opt. Express 5, 287-292 (2014).

5. M. A. Wallenburg et al., "Polarimetry-based method to extract geometry-independent metrics of tissue anisotropy," Opt. Lett. 35, 2570-2572 (2010).

6. Y. Gan and C. P. Fleming, "Extracting three-dimensional orientation and tractography of myofibers using optical coherence tomography," Biomed. Opt. Express 4(10), 2150-2165 (2013).

7. H. Wang, C. Lenglet, and T. Akkin, "Structure tensor analysis of serial optical coherence scanner images for mapping fiber orientations and tractography in the brain," J. Biomed. Opt. 20(3), 036003 (2015).

8. N. Ugryumova, S. V. Gangnus, and S. J. Matcher, "Three-dimensional optic axis determination using variable-incidence-angle polarizationoptical coherence tomography," Opt. Lett. 31(15), 2305-2307 (2006).

9. N. Ugryumova et al., "Novel optical imaging technique to determine the 3-D orientation of collagen fibers in cartilage: variable-incidence angle polarization-sensitive optical coherence tomography," Osteoarthr. Cartil. 17(1), 33-42 (2009).

10. C. E. Saxer et al., "High-speed fiber based polarization-sensitive optical coherence tomography of in vivo human skin," Opt. Lett. 25(18), 1355-1357 (2000).

11. M. K. Al-Qaisi and T. Akkin, "Polarization-sensitive optical coherence tomography based on polarization-maintaining fibers and frequency multiplexing," Opt. Express 16(17), 13032-13041 (2008).

12. H. Wang, J. Zhu, and T. Akkin, "Serial optical coherence scanner for large-scale brain imaging at microscopic resolution," Neurolmage $\mathbf{8 4}$, 1007-1017 (2014).

13. Z. Lu and S. J. Matcher, "Absolute fast axis determination using non-polarization- maintaining fiber-based polarization-sensitive optical coherence tomography," Opt. Lett. 37(11), 1931-1933 (2012).

14. H. Wang, M. K. Al-Qaisi, and T. Akkin, "Polarization-maintaining fiber based polarization-sensitive optical coherence tomography in spectral domain," Opt. Lett. 35(2), 154-156 (2010).

15. E. Götzinger, M. Pircher, and C. K. Hitzenberger, "High speed spectral domain polarization sensitive optical coherence tomography of the human retina," Opt. Express 13(25), 10217-10229 (2005).

16. E. Z. Zhang et al., "Numerical compensation of system polarization mode dispersion in polarization-sensitive optical coherence tomography," Opt. Express 21(1), 1163-1180 (2013). 